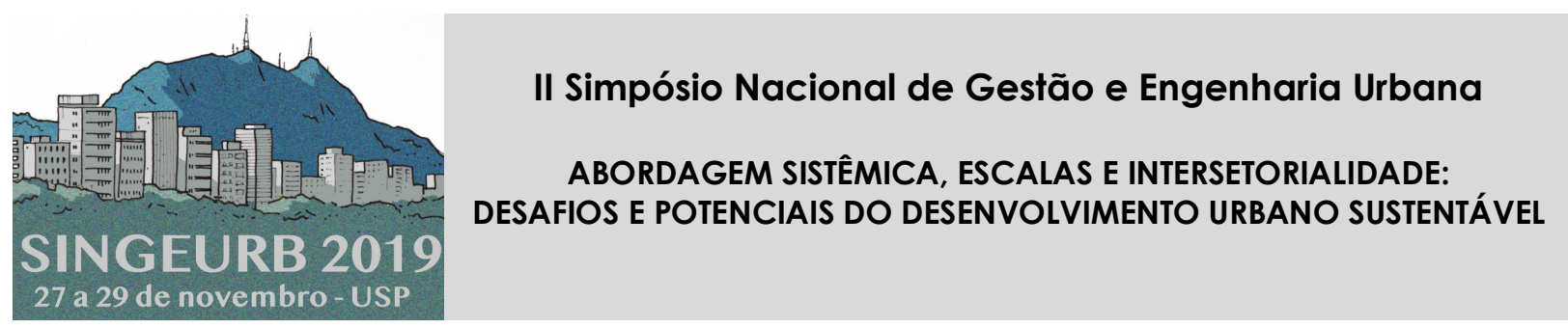

\title{
Macro e microplástico no sedimento fluvial na cidade de Itirapina/SP 1
}

\section{Macro and microplastic in the streambed sediment Itirapina city/SP}

\author{
Toyama, Daniele'; Menezes, Denise B. ${ }^{2}$; \\ ' Universidade Federal de São Carlos, Departamento de Engenharia Civil, \\ Programa de Pós-Graduação em Engenharia Urbana, São Carlos, São Paulo, \\ Brasil, danitoyama01@gmail.com \\ 2 Universidade Federal de São Carlos, denisebm@ufscar.br
}

\begin{abstract}
RESUMO
Estudos sobre antropização de sedimentos são recentes e escassos para água doce. 0 objetivo deste trabalho, que apresenta resultados parciais de uma pesquisa de mestrado, é apresentar uma análise do sedimento fluvial em córregos do município de Itirapina - SP, a fim de avaliar se está ocorrendo antropização. A metodologia consiste em coleta do sedimento, divisão da amostra em camadas e posteriormente em frações granulométricas e análise sob estereomicroscópio. Foi realizada a contagem do material tecnogênico encontrado nas amostras e constatou-se que ocorre a antropização do sedimento principalmente por macro e microplásticos. O córrego de referência não apresentou material tecnogênico em sua composição, enquanto os demais, localizados na área urbana, apresentaram. O ambiente lêntico apresenta um maior aporte de resíduos, em relação ao lótico, por ser uma zona de maior sedimentação do material oriundo da drenagem urbana. É necessário investir na gestão de resíduos sólidos para eliminar a carga desse material para a drenagem, que por consequência é transportado ao mar e causa diversos impactos negativos na biota. Além disso, o plástico é persistente no ambiente, sendo o principal tecnofóssil que as gerações futuras herdarão da atual.
\end{abstract}

Palavras-chave: Antropização, Resíduos Sólidos, Depósito Tecnogênico.

\section{ABSTRACT}

Studies about sedimentation are recent and scarce for freshwater. The objective of this work, which presents the partial results of a master's research, is to present an analysis of the streambed sediment in streams of the ltirapina city (SP), in order to evaluate if anthropization is occurring. The methodology consists of sediment collection, division of the sample into granulometric fractions and analysis under stereomicroscope. The technogenic material found in the samples was counted and it was verified that the anthropization of the sediment occurs mainly by macro and microplastics. The reference stream (blank) did not present technogenic material in its composition, while the rest, located in the urban area, presented. The lentic environment presents a greater contribution of residues, in relation to the lotic, because it is a

${ }^{1}$ TOYAMA, Daniele; MENEZES, Denise B. Antropização do sedimento fluvial em Itirapina - SP. In: II SIMPÓSIO NACIONAL DE GESTÃO E ENGENHARIA URBANA: SINGEURB, 2019, São Paulo. Anais... Porto Alegre: ANTAC, 2019. 
zone of greater sedimentation of the material coming from the drainage. It is necessary to invest in the management of solid waste to eliminate the load of this material for the drainage, which consequently is transported to the sea and causes several negative impacts on the biota. In addition, plastic is persistent in the environment, being the main technofossil that future generations will inherit from the current.

Keywords: Anthropization, Solid Waste, Technogenic deposit.

\section{INTRODUÇÃO}

A ocupação urbana provoca diversos problemas ambientais (e. g. áreas contaminadas, ocupações irregulares, desmatamento, degradação de áreas de várzea e da qualidade dos recursos hídricos, proliferação de doenças de veiculação hídrica). Um dos grandes desafios enfrentados atualmente é o gerenciamento dos resíduos sólidos, sendo comum a ocorrência de descartes irregulares em terrenos ociosos, ruas e às margens de córregos urbanos. A gestão precária faz com que esse material seja transportado aos rios, onde parte sedimenta, criando depósitos geológicos compostos por materiais artificialmente produzidos.

Esses depósitos são chamados de tecnogênicos e segundo Santos et al. (2017), no Brasil os estudos relacionados a eles vêm sendo realizados desde a década de 1990. Dentre as possibilidades de pesquisa em Geologia do Tecnógeno, o estudo dos sedimentos (POLETO et al., 2009; POLETO; MARTINEZ, 2011) é um campo de pesquisa recente e destaca-se a importância de avaliar como o ser humano está impactando a superfície terrestre e os corpos hídricos.

O plástico é o principal resíduo doméstico gerado em centros urbanos, sendo crescente o volume de sua utilização e as implicações ambientais inerentes ao seu descarte não racional pós-consumo (FORLIN; FARIA, 2002). Naturalmente, após seu descarte os resíduos plásticos vão se fragmentando em partículas menores ao longo do eixo longitudinal de deslocamento do rio. Parte desse material sedimenta no fundo e margens, e parte é transportada até o mar.

Os canais fluviais são um conduto deste material, existindo pouco conhecimento sobre abundância, padrões e características dos plásticos em água doce, sendo necessários estudos que permitam entender seu transporte e acúmulo nesse sistema (TIBBETTS et al., 2018; ZBYSZEWSKI; CORCORAN; HOCKIN, 2014; IMHOF et al., 2013; FREE et al., 2014; MANI et al., 2015; LECHNER et al., 2014). De acordo com Tibbets et al. (2018) atualmente não há estudos que quantifiquem a abundância de plásticos (micro) em ambientes bentônicos fluviais.

Assim sendo, este trabalho visa apresentar resultados parciais de pesquisa sobre a análise de sedimentos fluviais localizados em Itirapina (São Paulo) para identificar se está ocorrendo sedimentação de materiais de origem antrópica e consequente formação de depósitos tecnogênicos fluviais.

\section{METODOLOGIA}

\section{1 Área de estudo}

A bacia hidrográfica do córrego Água Branca (Figura 1) localiza-se no município de ltirapina - SP e abrange quase a totalidade da área urbana. A cidade possui população estimada em 17.589 habitantes e área territorial de 564,603 km² (IBGE, 2018). 99,75\% do município é atendido pela coleta de lixo e $88,99 \%$ das residências possui esgotamento sanitário (SEADE, 2018). A economia do município é predominantemente gerada pela prestação de serviços $(72,54 \%)$ (SEADE, 2018) e possui duas Unidades de Conservação - UC. A fitofisionomia do ambiente é Cerrado (Campo Sujo, Campo Limpo, Campo Cerrado e Campo Úmido, e fragmentos de Cerrado sensu stricto, Matas Galeria e Cerradão) (INSTITUTO FLORESTAL, 2017). A bacia é de terceira ordem, com $24 \mathrm{~km}$ de canais drenando a área de 40,3 km². 
Figura 1 - Localização da área de estudo

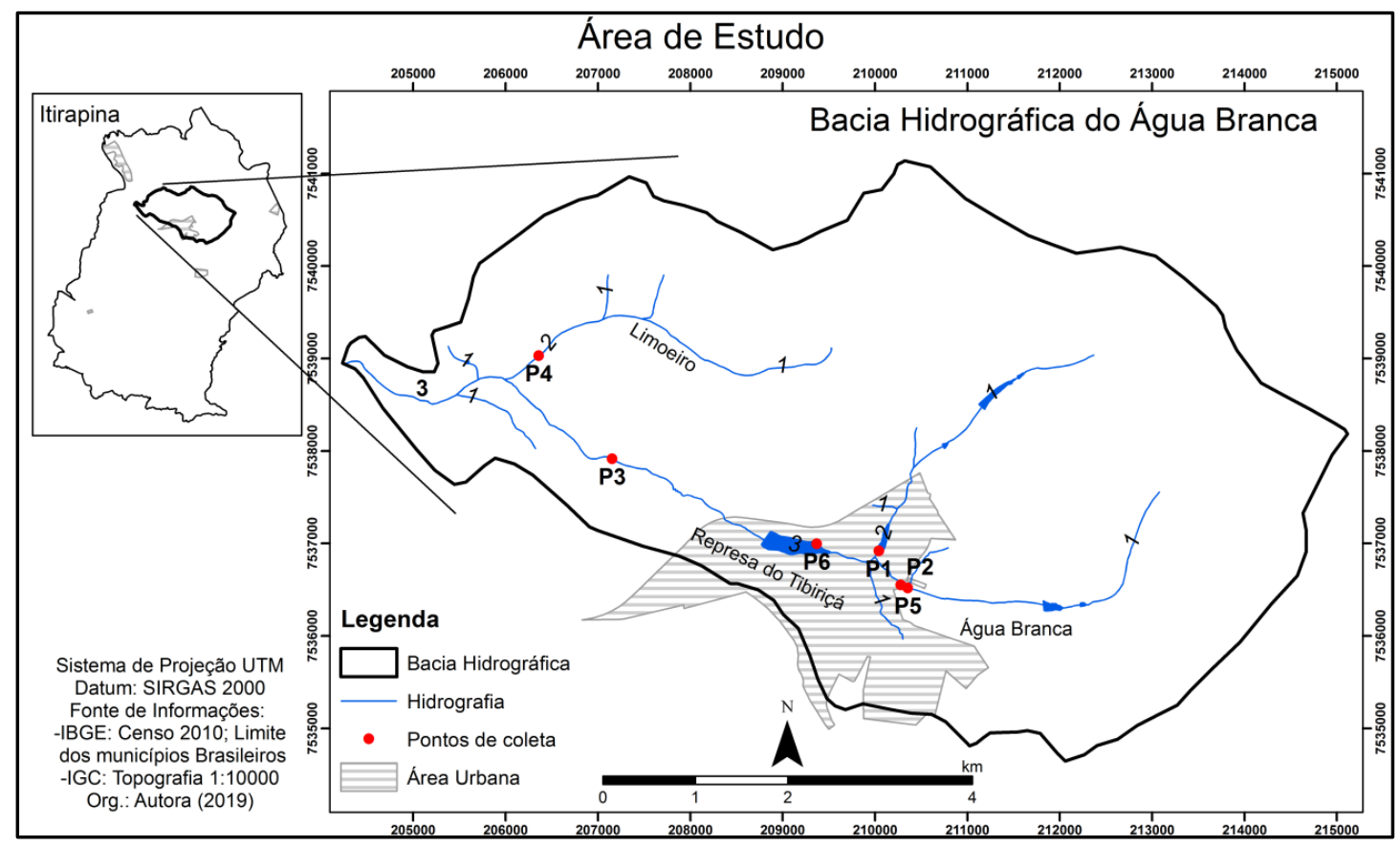

Fonte: $O$ autor (2019)

\subsection{Coleta das amostras}

As amostras foram coletadas em novembro de $2018 \mathrm{em}$ seis pontos (Figura 1; Quadro 1). 0 ponto de referência para as análises (P4) localiza-se dentro da Estação Experimental de Itirapina. Os demais pontos estão em áreas que sofrem maior ou menor influência da ocupação urbana. Foram amostradas as regiões de remanso dos córregos, onde há maior sedimentação (GOMES; FILIZOLA, 2006).

Quadro 1 - Descrição dos pontos de coleta

\begin{tabular}{|c|l|}
\hline Ponto & \multicolumn{1}{c|}{ Descrição } \\
\hline P1 & $\begin{array}{l}\text { Córrego Tibiriçá: sem mata ciliar, próximo à área urbana e à linha férrea. Na região há } \\
\text { deposição de resíduos sólidos. }\end{array}$ \\
\hline P2 & $\begin{array}{l}\text { Córrego Água Branca: sem vegetação ciliar, com intensa ocupação urbana nas duas } \\
\text { margens. }\end{array}$ \\
\hline P3 & $\begin{array}{l}\text { Córrego Água Branca: área com vegetação ciliar, localizado após destinação de } \\
\text { efluente da estação de tratamento de esgoto. }\end{array}$ \\
\hline P4 & $\begin{array}{l}\text { Córrego Limoeiro: área com vegetação ciliar e entorno ocupado pela produção de pinus } \\
\text { sp, não manejada há cinquenta anos. }\end{array}$ \\
\hline P5 & $\begin{array}{l}\text { Córrego Água Branca: sem vegetação ciliar, com entorno ocupado pela produção } \\
\text { agrícola familiar a montante da área urbana. }\end{array}$ \\
\hline P6 & \begin{tabular}{l} 
Represa do Tibiriçá: mudança de ambiente lótico para lêntico, com vegetação ciliar. \\
\hline
\end{tabular}
\end{tabular}

Fonte: $O$ autor (2019)

Foi utilizado um amostrador do tipo "corer", que retira um perfil de sedimento, confeccionado com tubo de PVC geomecânico de 2 polegadas e êmbolo interno (Figura 2). As amostras foram divididas de acordo com a mudança de coloração das camadas, indicativo de eventos de sedimentação distintos e foram armazenadas em sacos plásticos à temperatura ambiente e sem incidência de luz. 
Figura 2 - Modelo 3D do amostrador construído para este estudo. A: Manípulo; B: Manopla; C: Cap de PVC; D: Tubo de PVC rígido; E: Barra com rosca; F: Êmbolo

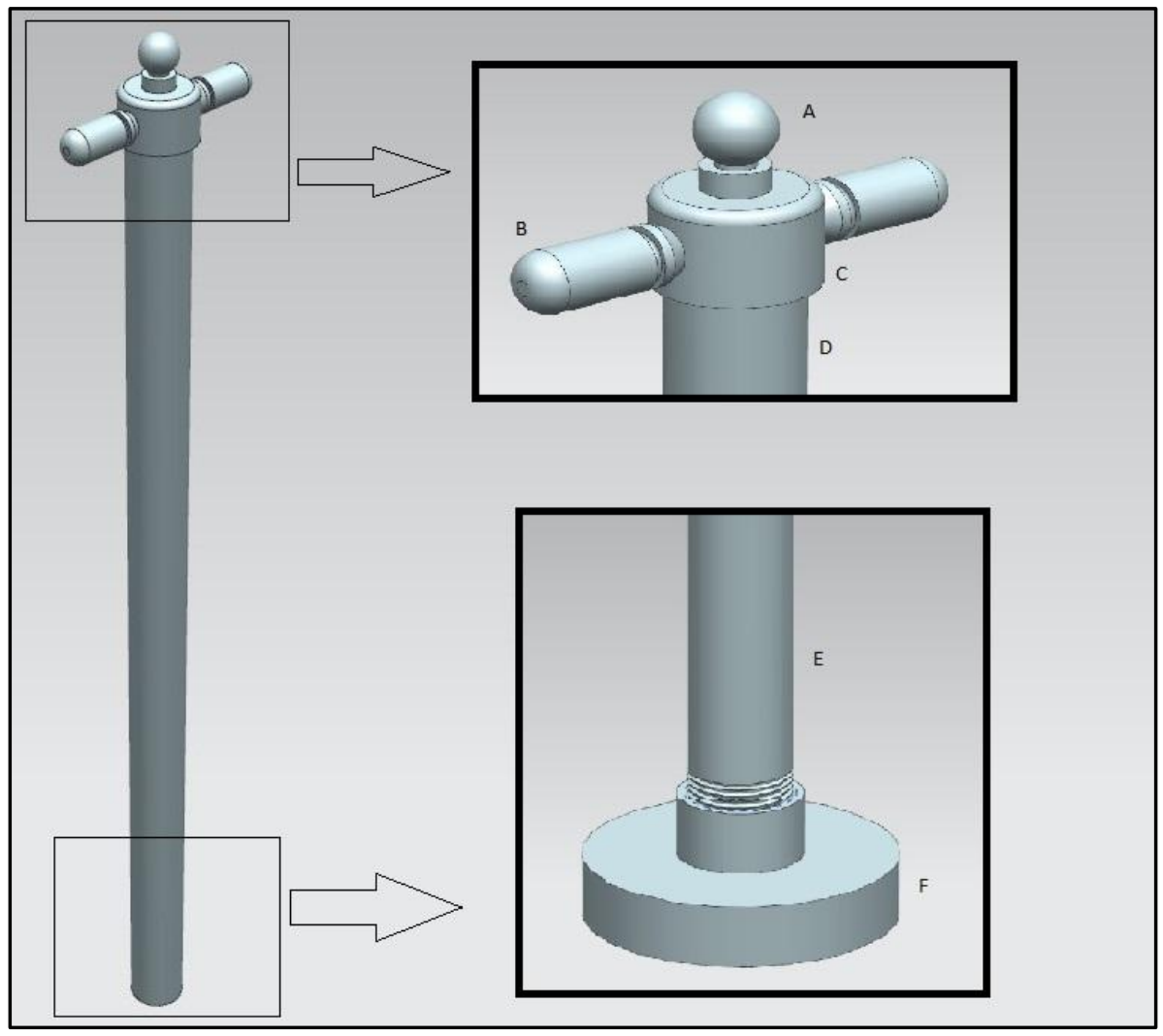

Fonte: Toyama, T. T. (2019)

\subsection{Preparo e análise das amostras}

As subamostras foram secas em temperatura ambiente e divididas em frações granulométricas ( $>4,75 \mathrm{~mm}, 4,75$ a 1,18 mm e 1,18 mm a $75 \mu \mathrm{m}$ ). As frações foram pesadas e em seguida, com o uso de uma lupa de mão, o material $>1,18 \mathrm{~mm}$ foi triado. $O$ material de $1,18 \mathrm{~mm}$ a $75 \mu \mathrm{m}$ foi quarteado e analisado sob estereomicroscópio. Quando encontrados, os resíduos de origem antrópica foram fotografados e separados.

\section{RESULTADOS E DISCUSSÃO}

Devido à heterogeneidade das amostras, foram analisados volumes distintos. A Tabela 1 apresenta a profundidade do perfil coletado (e suas subdivisões), o peso de cada fração e a quantidade de fragmentos de material não natural. 
Tabela 1 - Materiais não naturais encontrados na análise sob estereomicroscópio, de acordo com as camadas divididas. A posição 0 indica superfície, enquanto o valor crescente, a profundidade.

\begin{tabular}{|c|c|c|c|c|c|}
\hline $\begin{array}{c}\text { P1 } \\
\text { Profundidade/peso da } \\
\text { amostra }\end{array}$ & $\begin{array}{l}\mathrm{N}^{\circ} \\
\text { frag. }\end{array}$ & $\begin{array}{c}\text { P2 } \\
\begin{array}{c}\text { Profundidade/peso da } \\
\text { amostra }\end{array}\end{array}$ & $\begin{array}{l}\mathrm{N}^{\circ} \\
\text { frag. }\end{array}$ & $\begin{array}{c}\text { P3 } \\
\text { Profundidade/peso da } \\
\text { amostra }\end{array}$ & $\begin{array}{c}\mathrm{N}^{\circ} \\
\text { frag. }\end{array}$ \\
\hline $\begin{array}{c}\text { P1.1:0- } 7 \mathrm{~cm}(40,55 \\
\text { g) }\end{array}$ & 1 & $\begin{array}{c}\text { P2.1: } 0-7 \mathrm{~cm}(14,11 \\
\text { g) }\end{array}$ & 13 & $\begin{array}{c}\text { P3.1: } 0-7 \mathrm{~cm}(60,70 \\
\text { g) }\end{array}$ & 2 \\
\hline \multirow[t]{4}{*}{$\begin{array}{c}\text { P1.2: } 7-17 \mathrm{~cm}(45,43 \\
\text { g) }\end{array}$} & 0 & $\begin{array}{c}\text { P2.2: } 7-16 \mathrm{~cm}(18,78 \\
\mathrm{g})\end{array}$ & 6 & $\begin{array}{c}\text { P3.2: } 7 \text { - } 10 \mathrm{~cm}(21,68 \\
\text { g) }\end{array}$ & 0 \\
\hline & & $\begin{array}{c}\text { P2.3: } 16-20 \mathrm{~cm} \\
(37,66)\end{array}$ & 19 & $\begin{array}{c}\text { P3.3: } 10-14 \mathrm{~cm}(31,4 \\
\mathrm{g})\end{array}$ & 0 \\
\hline & & & & $\begin{array}{c}\text { P3.4:14 - } 18 \mathrm{~cm} \\
(22,19 \mathrm{~g})\end{array}$ & 0 \\
\hline & & & & $\begin{array}{c}\text { P3.5: } 18-26 \mathrm{~cm} \\
(59,79 \mathrm{~g})\end{array}$ & 2 \\
\hline $\begin{array}{c}\text { P4 } \\
\begin{array}{c}\text { Profundidade/peso da } \\
\text { amostra }\end{array}\end{array}$ & $\begin{array}{c}\mathbf{N}^{\circ} \\
\text { frag. }\end{array}$ & $\begin{array}{c}\text { P5 } \\
\text { Profundidade/peso da } \\
\text { amostra }\end{array}$ & $\begin{array}{c}\mathbf{N}^{\circ} \\
\text { frag. }\end{array}$ & $\begin{array}{c}\text { P6 } \\
\text { Profundidade/peso da } \\
\text { amostra }\end{array}$ & $\begin{array}{c}\mathbf{N}^{\circ} \\
\text { frag. }\end{array}$ \\
\hline $\begin{array}{c}\text { P4.1: } 0-8 \mathrm{~cm}(59,77 \\
\text { g) }\end{array}$ & 0 & P5.1:0 - $6 \mathrm{~cm}(9,67 \mathrm{~g})$ & 4 & $\begin{array}{c}\text { P6.1: } 0-5 \mathrm{~cm}(0,89 \\
\text { g) }\end{array}$ & 8 \\
\hline $\begin{array}{c}\text { P4.2: } 8-15 \mathrm{~cm}(31,94 \\
\text { g) }\end{array}$ & 0 & $\begin{array}{c}\text { P5.2: } 6-11 \mathrm{~cm}(16,76 \\
\mathrm{g})\end{array}$ & 2 & $\begin{array}{c}\text { P6.2: } 5-11 \mathrm{~cm}(22,33 \\
\text { g) }\end{array}$ & 6 \\
\hline \multirow[t]{2}{*}{$\begin{array}{c}\text { P4.3: } 15-25 \mathrm{~cm}(78,8 \\
\mathrm{g})\end{array}$} & 0 & $\begin{array}{l}\text { P5.3: } 11-18 \mathrm{~cm} \\
(34,65 \mathrm{~g})\end{array}$ & 0 & $\begin{array}{c}\text { P6.3: } 11-22 \mathrm{~cm} \\
(62,18 \mathrm{~g})\end{array}$ & 8 \\
\hline & & & & $\begin{array}{c}\text { P6.4: } 22-32 \mathrm{~cm} \\
(28,65 \mathrm{~g})\end{array}$ & 1 \\
\hline
\end{tabular}

Fonte: O autor (2019)

O principal resíduo encontrado foi o plástico (macro e micro). O microplástico pode ser inserido no ambiente aquático a partir da degradação do macroplástico ou inserção de micropartículas artificiais a partir de atividades humanas (KLEIN et al., 2018). No P6 - Represa do Tibiriçá, foram encontrados macroplásticos, sendo um fragmento maior que $4,75 \mathrm{~mm}$ na camada de 0 a $5 \mathrm{~cm}$; um fragmento maior que $4,75 \mathrm{~mm}$ e 4 fragmentos maiores que $1,18 \mathrm{~mm}$, na camada de 5 a $11 \mathrm{~cm}$ de profundidade (Figura 3).

Os microfragmentos triados são em sua maioria microplásticos, fibras de tecidos sintéticos, vidro e papel alumínio (Figura 3). O P2 (área de intensa ocupação urbana) e o P6 (represa) foram os locais com maior presença de resíduos. No P6, por ser um ambiente lêntico, há um grande acúmulo de resíduos (Figura 4). Em observação de campo foi encontrado vasilhame datado de 1991, indicando acumulação há décadas e formando depósitos tecnogênicos. Alguns hotspots de acúmulo de microplásticos são lagos, várzeas e meandros (ambientes de baixa velocidade da água) que podem apresentar maior acumulação de materiais do que o próprio canal (TIBBETTS et al., 2018).

No ponto de referência (P4) os resíduos oriundos das atividades humanas são ausentes, mostrando que ocupação urbana está degradando o sedimento dos corpos hídricos. Isso causa diversos problemas, uma vez que animais podem consumir esse material para alimentação ou construção de casulos. Há relatos do consumo do microplástico por peixes (KHAN et al., 2018), que por consequência são consumidos por humanos.

Segundo Klein et al. (2018) a degradação do plástico no ambiente aquático é lenta se comparada com a degradação desse material exposto na litosfera, fazendo com que o plástico persista na hidrosfera por centenas de anos, sendo considerado o principal tipo de tecnofóssil (ZALASIEWICZ et al., 2016). 
Figura 3 - Fragmentos observados no sedimento. A: Fragmento de vidro; B: Fibra; C: Fibra aderida em borracha; D: Microplástico; E: Papel Alumínio; F: Microplástico; G e H: Macroplástico

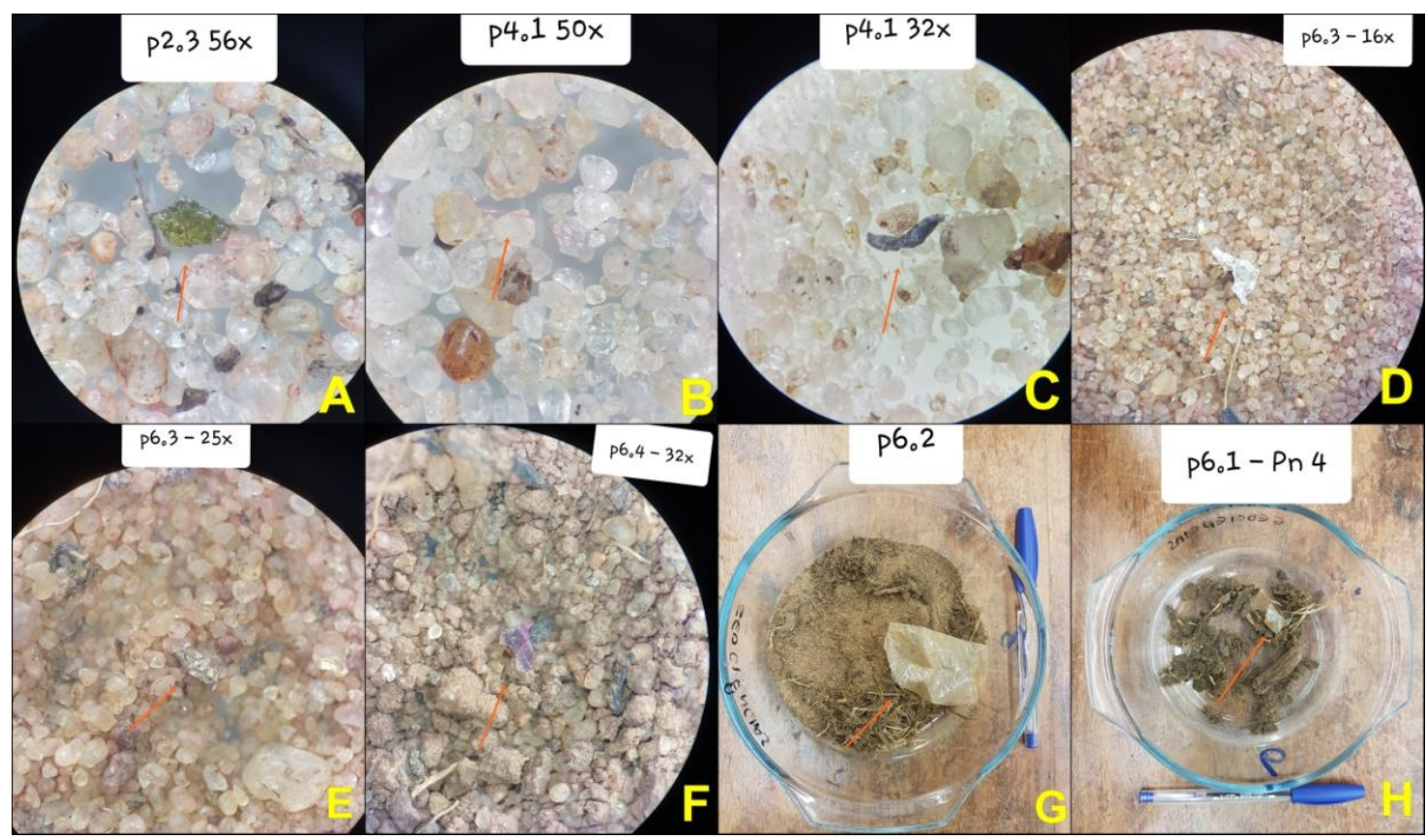

Fonte: $\bigcirc$ autor (2019)

Figura 4 - Plásticos acumulados na Represa do Tibiriçá

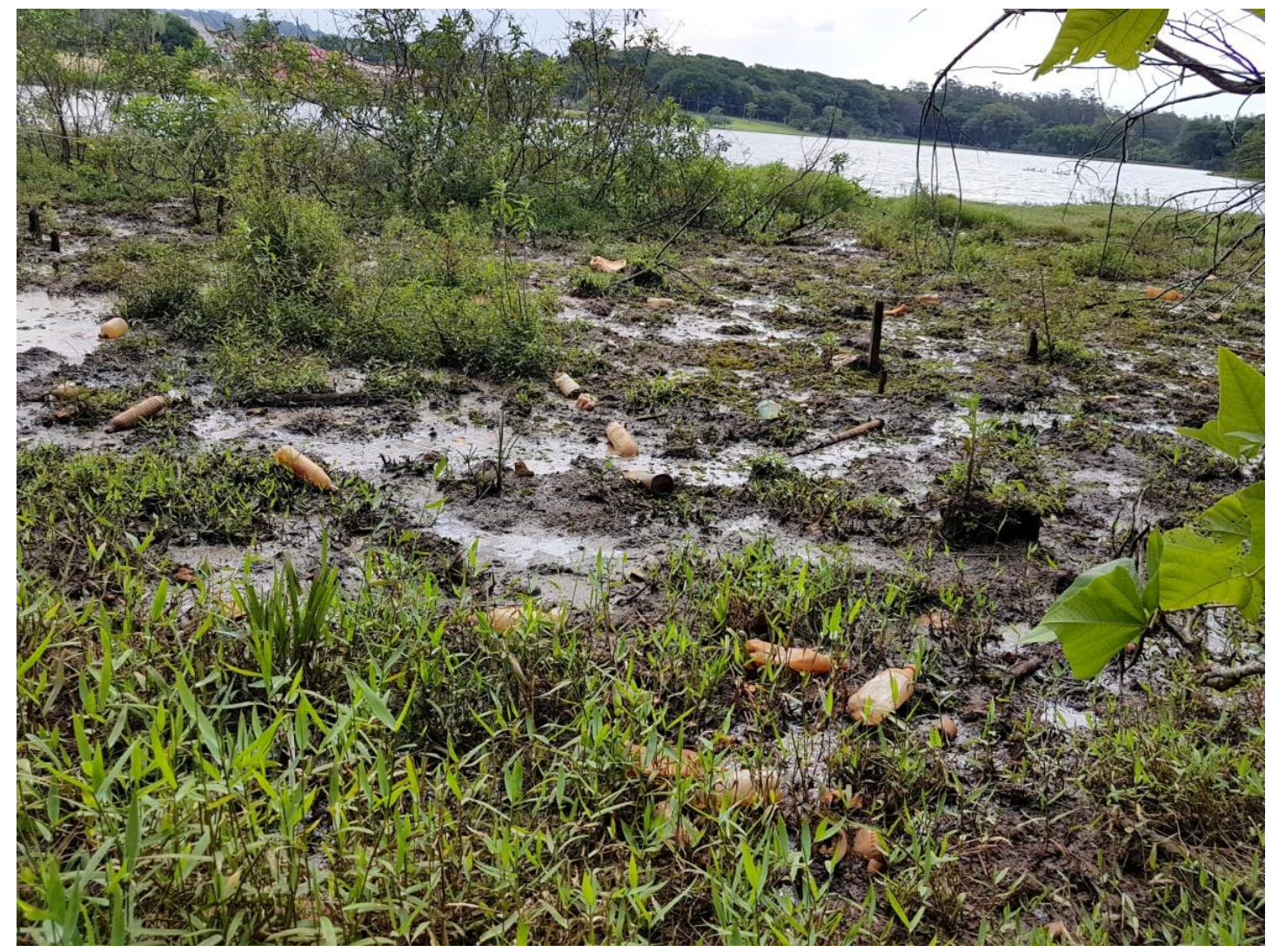

Fonte: $O$ autor (2019)

Por ser de densidade menor que a água, rochas e minerais, o plástico é facilmente transportável, viajando rapidamente e por longas distâncias pela Terra através da água 
(ZALASIEWICZ; GABBOTT; WATERS, 2019); sua sedimentação ocorre quando sua densidade torna-se maior que a da água. Isso pode ocorrer através da associação do plástico com algas, organismos ou sedimento (ZALASIEWICZ; GABBOT; WATERS, 2019).

No ambiente urbano, uma fonte de microplásticos na água são as estações de tratamento de esgoto - ETE (TIBBETTS et al., 2018; KAY et al., 2018). O efluente de uma ETE que atende uma população de 650 mil habitantes pode ter aproximadamente 65 milhões de partículas microplásticas (MURPHY et al., 2016). O efluente doméstico também carrega uma grande carga de microplásticos (fibras oriundas da lavagem de tecidos) (NAPPER; THOMPSON, 2016), aumentando no inverno (BROWNE et al., 2011).

Apesar de o P3 localizar-se após a ETE, não há grande sedimentação de fragmentos. $O$ mesmo ocorre na Estação Experimental que possui mata ciliar, não recebendo descargas diretas do uso urbano. No Pl também é pouco expressivo, uma vez que o uso urbano é menos intenso e no P5 a presença de fragmentos é intermediária, uma vez que às margens há produção agrícola familiar e possui influência urbana moderada.

\section{CONCLUSÕES}

Podemos concluir que os sedimentos fluviais de Itirapina estão sofrendo o processo de antropização, sendo mais expressivo na área de intensa ocupação urbana e na represa. As principais fontes desse material é o descarte inapropriado de resíduos nas ruas ou margem dos rios, que com o regime de chuvas é levado até o canal. Destacamos a necessidade de se investir no gerenciamento de resíduos e medidas estruturais para evitar que esse material alcance os rios. Além da degradação do ambiente natural, esse material pode contaminar peixes que posteriormente serão consumidos pelo homem. Algumas ações que podem reduzir a carga dos materiais de origem antrópica é o investimento em políticas de educação ambiental, reciclagem e descarte final adequado dos resíduos, inserir dispositivos separadores de fluxos no sistema de drenagem e promover o tratamento do primeiro fluxo de água das chuvas.

\section{AGRADECIMENTOS}

O presente trabalho foi realizado com apoio da Coordenação de Aperfeiçoamento de Pessoal de Nível Superior - Brasil (CAPES) - Código de Financiamento 001.

\section{REFERÊNCIAS}

BROWNE, M. A. et al. Accumulation of Microplastic on Shorelines Woldwide: Sources and Sinks. Environmental Science \& Technology. v.45. p. 9175-9179. 2011.

FORLIN, F. J.; FARIA, J. A. Considerações sobre a reciclagem de embalagens plásticas. Polímeros: Ciência e Tecnologia. v. 12, n.1, p. 1-10. 2002.

FREE, C.M. et al. High-levels of microplastic pollution in a large, remote, mountain lake. Mar. Pollut. Bull. 2014, 85, 156-163.

GOMES, M. A. F.; FILIZOLA, H. F. Amostragem de sedimentos para análise de metais pesados. Cap. 10. In: Manual de procedimentos de coleta de amostras em áreas agrícolas para análise de qualidade ambiental: solo, água e sedimentos. Embrapa Meio Ambiente. 169 p. 2006.

IBGE - INSTITUTO BRASILEIRO DE GEOGRAFIA E ESTATÍSTICA. Cidades: Itirapina. 2009. Disponível em: < https://cidades.ibge.gov.br/brasil/sp/itirapina/panorama>. Acesso em abril de 2018.

IMHOF, H. K. et al. Contamination of beachsediments of a subalpine lake with microplastic particles. CurrBiol. v. 23, n. 19. p. 867-868. 2013. 
INSTITUTO FLORESTAL. Estações Ecológica e Experimental de Itirapina. 2017. Disponível em: <http://www.iflorestal.sp.gov.br/ltirapina/>. Acesso em março de 2018.

KAY, P. et al. Wastewater treatment plants as a source of microplastics in river catchments. Environmental Science and Pollution Research. v. 25, n. 20. p. 20264-20267. 2018.

KHAN, F. R. et al. Microplastics in inland African waters: presence, sources and fate. In: Wagner M.; Lambert S. FreshwaterMicroplastics: Emerging Environmental Contaminants? Springer, Heidelberg. 2018.

KLEIN, S. et al. Analysis, Occurrence, and Degradation of Microplastics in the Aqueous Environment. FreshwaterMicroplastics. p. 51 - 67. 2018.

LECHNER, A. et al. The Danube so colourful: a potpourri of plastic litter out numbers fish larvae in Europe's second largest river. EnvironPollut. v. 188. p. 177-181. 2014.

MANI, T. et al. Microplastics profile along the Rhine River. Sci. Rep. v. 5. 2015.

MURPHY, F. et al. Wastewater Treatment Works (WWTW) as a Source of Microplastics in the Aquatic Environment. Environmental Science \& Technology. v. 50, n. 11. p. 5800-5808. 2016.

NAPPER, I. E.; THOMPSON, R. C. Release of synthetic microplastic plastic fibres from domestic washing machines: Effects of fabric type and washing conditions. Marine PollutionBulletin. $v$. 112 , n. 1-2. p. 39-45. 2016.

POLETO, C. et al. Urban Sediment Particle Size and Pollutants in Southern Brazil. Journal of Soils and Sediments. v. 17, n. 9, p. 317-327. 2009.

POLETO, C.; MARTINEZ, L. L. G. Sedimentos urbanos: ambiente e água. HOLOS Environment. v. 11, n. 1, p. 1-15. 2011.

POLETO, C.; MARTINEZ, L. L. G. Sedimentos urbanos: ambiente e água. HOLOS Environment. v. 11, n. 1, p. 1-15. 2011.

SANTOS, E. Q. G. et al. Terrenos e processos tecnogênicos na Área de Proteção Ambiental Cabuçu - Tanque Grande, Guarulhos (SP): análise, mapeamento e quantificação. Revista Brasileira de Geomorfologia. São Paulo. v. 18. n. 4, p. 825-839, outubro-dezembro. 2017. Disponível em: < http://www.lsie.unb.br/rbg/index.php/rbg/article/view/1279 >. Acesso em abril de 2018.

SEADE - FUNDAÇÃO SISTEMA ESTAUDUAL DE ANÁLISE DE DADOS. Perfil dos municípios paulistas: Itirapina. Disponível em: <http://www.perfil.seade.gov.br/? >. Acesso em abril de 2018.

TIBBETTS, J. et al. Abundance, Distribution, and Drivers of Microplastic Contamination in Urban River Environments. Water. v. 10, n. 11. 14 p. 2018.

ZALASIEWICZ, J. et al. The geological cycle of plastics and their use as a stratigraphic indicator of the Anthropocene. Anthropocene. v. 13, p. 4-17, março. 2016. Disponivel em: < https://www.sciencedirect.com/science/article/pii/S2213305416300029?via\%3Dihub> Acesso em abril de 2018.

ZALASIEWICZ, J.; GABBOTT, S.; WATERS, C. Plastic Waste: How Plastics Have Become Part of the Earth's Geological Cycle. In: Waste. Elsevier. 2019. 
ZBYSZEWSKI, M.; CORCORAN, P.L.; HOCKIN, A. Comparison of the distribution and degradation of plastic debris along shorelines of the Great Lakes, North America. J. Great Lakes Res. 2014, 40, 288-299. 